\title{
Years of Potential Life Lost and Productivity Costs Due to Premature Cancer-Related Mortality in Iran
}

\author{
Soheila Khorasani ${ }^{1}$, Satar Rezaei ${ }^{2}$, Hamideh Rashidian ${ }^{3}$, Rajabali Daroudi ${ }^{1 *}$
}

\begin{abstract}
Background: Cancer is recently one of the major concerns of the public health both in the world and Iran. To inform priorities for cancer control, this study estimated years of potential life lost (YPLL) and productivity losses due to cancer-related premature mortality in Iran in 2012. Materials and Methods: The number of cancer deaths by sex for all cancers and the ten leading causes of cancer deaths in Iran in 2012 were obtained from the GLOBOCAN database. The life expectancy method and the human capital approach were used to estimate the YPLL and the value of productivity lost due to cancer-related premature mortality. Results: There were 53,350 cancer-related deaths in Iran. We estimated that these cancer deaths resulted in 1,112,680 YPLL in total, 563,332 $(50.6 \%)$ in males and $549,348(49.4 \%)$ in females. The top 10 ranked cancers accounted for $75 \%$ of total death and $70 \%$ of total YPLL in the males and $69 \%$ for both death and YPLL in the females. The largest contributors for YPLL in the two genders were stomach and breast cancers, respectively. The total cost of lost productivity due to cancer-related premature mortality discounted at 3\% rate in Iran, was US\$1.93 billion. The most costly cancer for the males was stomach, while for the females it was breast cancer. The percentage of the total costs that were attributable to the top 10 cancers was $67 \%$ in the males and $71 \%$ in the females. Conclusions: The YPLL and productivity losses due to cancer-related premature mortality are substantial in Iran. Setting resource allocation priorities to cancers that occur in younger working-age individuals (such as brain and central nervous system) and/or cancers with high incidence and mortality rates (such as stomach and breast) could potentially decrease the productivity losses and the YPLL to a great extent in Iran.
\end{abstract}

Keywords: Years of potential life lost - productivity costs - cancer - economic burden - Iran.

Asian Pac J Cancer Prev, 16 (5), 1845-1850

\section{Introduction}

Cancer is generally regarded as one of the most important causes of the deaths in the world. According to the GLOBOCAN database about 8.2 million people have died from cancer in 2012 (Ferlay et al., 2012). Cancer is recently one of the major concerns of the public health in Iran and is the third leading cause of death after coronary heart disease and road traffic injuries (Naghavi et al., 2009; Abachizadeh et al., 2011). In recent years incidence and mortality rate of cancer has decreased in high-income countries (HICs) due to preventive and therapeutic interventions, but in low and middle-income countries (LMCs) such as Iran, it has remarkably increased (Jemal et al., 2010; D'Souza et al., 2013; Roya and Abbas, 2013). It is estimated that the number of deaths from cancer in Iran will increase from 53,350 cases in 2012 to 58,087 in 2015 (Ferlay et al., 2012) (1). Therefore, it is necessary to conduct the preventive and therapeutic interventions to reduce the incidence and mortality of cancer in LMCs including Iran.
Due to resource limitation, it is important for the policymakers to prioritize the cancer control interventions (Kim et al., 2014). One of the useful indices in this way is the specific burden of each cancer. Several measures have been proposed to quantify the burden of cancer, some of which include: mortality rate, Years of Potential Life Lost (YPLL) and productivity loss (Ekwueme et al., 2011). Mortality rate cannot differentiate between the young and old ages deaths, in other words it does not show the burden of early deaths correctly. One of the known useful indexes quantifying the burden of cancer is the years of potential life lost (YPLL) (Burnet et al., 2005). This index measures the years of life lost due to premature death from special diseases, like cancer, in a population. Definition of premature death is conventional and different, for example it can be death at the ages less than 70 or 75 years or less than the average life expectancy. According to this definition, the deaths in younger age, causes more loss of life years than the deaths in later ages (Gardner and Sanborn, 1990; Lee, 1997).

There is a consensus that the economic burden of

${ }^{I}$ Department of Health Economics and Management, School of Public Health, Tehran University of Medical Sciences, Tehran, ${ }^{2}$ School of Public Health, Kermanshah University of Medical Sciences, Kermanshah, ${ }^{3}$ Biostatistics and Epidemiology Department, Kerman University of Medical Sciences, Kerman, Iran*For correspondence: rdaroudi@yahoo.com,daroudi@razi.tums.ac.ir 
Soheila Khorasani et al

cancer is substantial and one of the main elements of this economic burden is the cost of productivity lost due to cancer-related premature mortality (Bradley et al., 2008; Hanly et al., 2014). Recent studies reported that about 70 to $85 \%$ of economic burden of cancers is related to cost of productivity lost (Jonsson and Wilking, 2007). Assessing the economic burden of cancer provide useful information and valuable insights for decision makers to better resource allocation (Bradley et al., 2008). Although a few studies have estimated the direct costs of cancer in Iran (Akbarzadeh et al., 2008; Bazyar et al., 2010; Davari et al., 2013), to the best of our knowledge, no study quantifies the costs of cancer-related premature mortality in Iran at the national level. However, the aim of this study was to estimate the YPLL and cost of productivity lost from cancer-related premature mortality in Iran for the year of 2012 .

\section{Materials and Methods}

\section{General approach}

We used three broad measures of the disease burden to quantify the impact of cancer mortality in Iran in 2012: mortality number, YPLL, and the value of lost productivity due to premature mortality. The study extracted the number of cancer deaths in 2012 by sex for all cancers and the ten leading causes of cancer deaths in Iran, thus by these, it calculated the YPLL using the life expectancy method. It used the human capital approach to estimate the value of productivity lost due to cancer-related premature mortality.

\section{Data sources}

Numbers of cancer deaths by sex and age groups including 0-14, 15-39, 40-44, 45-49, 50-54, 55-59, 60-64, 65-69, 70-74, and 75+ were obtained from the GLOBOCAN 2012 website (Ferlay et al., 2012). Data were abstracted on the following cancer sites: oesophagus (ICD10 C15, males and females); stomach (C16, males and females); colorectal (C18-21, males and females); Liver (C22, males and females); lung (C33-34, males and females); breast (C50, females); ovary (C56 - females); prostate (C61, males); bladder (C67, males and females); brain and CNS (C70-72, males and females); nonHodgkin's lymphoma (C82-85, C96, males and females); and Leukemia (C91-95, males and females). The study also obtained the 2012 Iran life table from the World Health Organization (World Health Organization, 2014) to estimate the average life expectancy for each age group.

Data on age- and gender-specific wages came from the Iranian Ministry of Cooperation Labor and Social Welfare (Ministry of labor and social Welfare, 2013). There weren't any data about wages for the household activity, so the study used the minimum daily wage approved by the Iranian Ministry of Cooperation, Labor and Social Welfare in 2012 for the female household activities. Data on age and sex-specific employment and housekeeping rate in 2012 were extracted from the Iranian Statistical

Table 1. Number of Deaths and YPLL for the 10 most Common Males and Females Cancers and all Cancers in Iran, 2012

\begin{tabular}{|c|c|c|c|c|c|c|}
\hline \multirow[t]{2}{*}{ ICD-10 } & \multirow{2}{*}{ Cancer site } & \multicolumn{2}{|c|}{ Death } & \multicolumn{2}{|c|}{ YPLL } & \multirow[b]{2}{*}{$\begin{array}{c}\text { YPLL/ } \\
\text { death }\end{array}$} \\
\hline & & All deaths & $\begin{array}{c}\% \text { of the } \\
\text { total }\end{array}$ & Number & $\begin{array}{c}\% \text { of the } \\
\text { total }\end{array}$ & \\
\hline \multicolumn{7}{|c|}{ Males } \\
\hline \multirow[t]{2}{*}{ C00-97/C44 } & All cancers excl. non-melanoma skin cancer & 30,115 & 100.00 & 563,332 & 100.00 & 18.71 \\
\hline & Top 10 cancers & 22,574 & 74.96 & 396,874 & 70.45 & $\mathbf{1 7 . 5 8}$ \\
\hline C15 & Oesophagus & 2,662 & 8.84 & 43,802 & 7.78 & 16.45 \\
\hline $\mathrm{C} 16$ & Stomach & 5,665 & 18.81 & 84,426 & 14.99 & 14.90 \\
\hline C18-21 & Colorectal & 2,267 & 7.53 & 44,150 & 7.84 & 19.48 \\
\hline $\mathrm{C} 22$ & Liver & 847 & 2.81 & 15,407 & 2.73 & 18.19 \\
\hline C33-34 & Lung & 2,950 & 9.80 & 49,194 & 8.73 & 16.68 \\
\hline C61 & Prostate & 2,297 & 7.63 & 23,708 & 4.21 & 10.32 \\
\hline C67 & Bladder & 1,827 & 6.07 & 25,704 & 4.56 & 14.07 \\
\hline C70-72 & Brain and $\mathrm{CNS}$ & 1,021 & 3.39 & 31,393 & 5.57 & 30.75 \\
\hline C82-85,C96 & Non-Hodgkin lymphoma & 1,216 & 4.04 & 27,584 & 4.90 & 22.68 \\
\hline C91-95 & Leukemia & 1,822 & 6.05 & 51,506 & 9.14 & 28.27 \\
\hline \multicolumn{7}{|c|}{ Females } \\
\hline \multirow[t]{2}{*}{ C00-97/C44 } & All cancers excl. non-melanoma skin cancer & 23,235 & 100.00 & 549,348 & 100.00 & 23.64 \\
\hline & Top 10 cancers & 16,102 & 69.30 & 381,406 & 69.43 & 23.69 \\
\hline $\mathrm{C} 15$ & oesophagus & 2,253 & 9.70 & 41,903 & 7.63 & 18.60 \\
\hline C16 & Stomach & 2,582 & 11.11 & 46,097 & 8.39 & 17.85 \\
\hline C18-21 & Colorectal & 1,995 & 8.59 & 44,656 & 8.13 & 22.38 \\
\hline $\mathrm{C} 22$ & Liver & 645 & 2.78 & 12,102 & 2.20 & 18.76 \\
\hline C33-34 & Lung & 1,411 & 6.07 & 28,717 & 5.23 & 20.35 \\
\hline $\mathrm{C} 50$ & Breast & 3,304 & 14.22 & 94,216 & 17.15 & 28.52 \\
\hline C56 & Ovary & 1,076 & 4.63 & 26,430 & 4.81 & 24.56 \\
\hline C70-72 & Brain and CNS & 823 & 3.54 & 27,767 & 5.05 & 33.74 \\
\hline C82-85,C96 & Non-Hodgkin lymphoma & 771 & 3.32 & 18,542 & 3.38 & 24.05 \\
\hline C91-95 & Leukemia & 1,242 & 5.35 & 40,976 & 7.46 & 32.99 \\
\hline
\end{tabular}

*Bold refer to the sum aggregated totals for 'all cancers' and the 'top 10 cancers' ranked in Iran by mortality. 
Center (SCI, 2013).

\section{Estimation methods}

YPLL estimation: to estimate the YPLL, the study assumed all deaths in each age group occurred in the mid-point of that age group then the number of deaths in each age group were multiplied by the corresponding average remaining life expectancy obtained from the 2012 Iran life tables. The estimated number of the YPLL in each age group was summed to obtain the total YPLL for all cancers, and the ten leading cancer in the males and females. In order to calculate the average YPLL per death, the value of YPLL was divided by the number of death.

Premature mortality cost estimation: The human capital approach assumes that an individual produces a stream of earnings over a working lifetime which is cut short by premature death (Hanly et al., 2015). In order to estimate the premature mortality costs for each cancerrelated death, the study disregarded any death below 15 years, and then the YPLL (resulted from death $\geq 15$ years) was multiplied by age and gender-specific annual wages from the age of death until the expected life. Estimates were adjusted for the employment and housekeeping rate. The study assumed a fixed wage rate for the future years; future costs were discounted at $3 \%$ per annum. To obtain the total costs for every age group, the estimated costs were summed over deaths in each age group. Afterwards, total costs for each cancer type and all cancers combined were estimated through the summation of costs for the all age groups. It also expressed premature mortality costs per cancer death and per YPLL. All the costs were converted to US dollars (US \$) using the average annual 2012 exchange rate (US\$1=Rial 12,290) (CBI, 2014).

\section{Results}

According to the GLOBOCAN 2012 estimates, there were 53,350 cancer-related death in Iran which 30,115 $(56.4 \%)$ of them were in the males. The study estimated that these cancer deaths resulted in 1,112,680 YPLL in total, 563,332 (50.6\%) in the males and 549,348 (49.4\%) in the females. The top 10 ranked cancers accounted for $75 \%$ of total death and $70 \%$ of total YPLL in the males and $69 \%$ for both death and YPLL in the females (Table 1).

The most common cause of cancer-related death was stomach in the males, whereas it was breast in the females. Also, the largest contributor for the YPLL in the males and females was stomach and breast cancers, respectively. The YPLL ranking for the most of cancers were different from their rankings according to the numbers of deaths. For example, Leukemia cancer in the males ranked 7th in terms of deaths, while this cancer ranked 2nd in terms of the YPLL (Table 1).

The average of YPLL per cancer death was 18.71 in the males, while it was 23.64 in the females. Among the top 10 sites, Brain and CNS cancers in the males had the highest average number of YPLL per death (30.75) and prostate cancer had the lowest (10.32). Brain and CNS cancers in the females had as in the males the highest average number of YPLL per death (33.74), but stomach cancer had the lowest (17.85) (Table 1).
Figure 1 shows the YPLL for the ten leading causes of cancer deaths by sex in Iran. For the combined males and females, the highest number of the YPLL was related to stomach cancer, followed by breast and Leukemia cancers, respectively. In addition, the lowest number of YPLL was associated with prostate and bladder cancers, respectively.

The total cost of lost productivity due to cancer-related premature mortality discounted at 3\% rate, in 2012 for Iran was US\$ 1.93 billion. The productivity costs were higher for the males than females $(\$ 1,209$ million $v$ s. $\$ 722$ million; Table 2). In terms of productivity costs, the most costly cancer for the males was stomach and accounted for $13 \%$ of the overall male cancer costs, while for the females it was breast cancer and accounted for $21 \%$ of the overall female cancer costs. The percentage of the total costs that were attributable to the top 10 cancers was $67 \%$ in the males and $71 \%$ in the females (Table 2).

The average premature mortality cost per cancer death was $\$ 40,946$ for males, while it was $\$ 31,720$ for females. Among the top 10 sites, the most and the least costly cancers per death in the males were Brain and CNS $(\$ 90,103$ per death) and prostate cancer $(\$ 7,161)$, respectively. Brain and CNS cancers in the females were as in the males the most costly cancers when expressed in terms of cost per death $(\$ 49,553)$, but the stomach cancer was the least costly $(\$ 20,838)$. For all combined cancers, the cost per YPLL was $\$ 2,313$ for males, while for females it was $\$ 1,402$. The cost per YPLL for males varied between \$694 (Prostate) and \$3,216 (Brainand CNS), for females it was between $\$ 1,170$ (Stomach) and $\$ 1,621$ (Brainand CNS) (Table 2).

Premature mortality costs (US \$ million) for the 10 most common cancers in both sexes are shown in figure 2 . In both sexes combined, the most costly cancer was

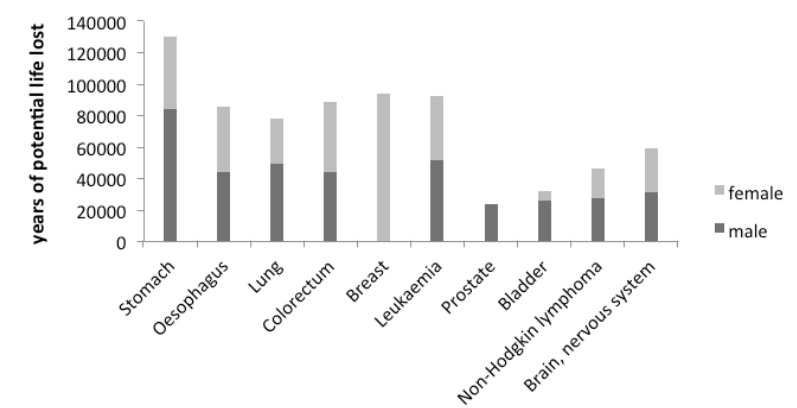

Figure 1. Years of Potential Life Lost (YPLL) for the 10 Most Common Cancers in Both Sexes (Ranked by Mortality), in Iran 2012

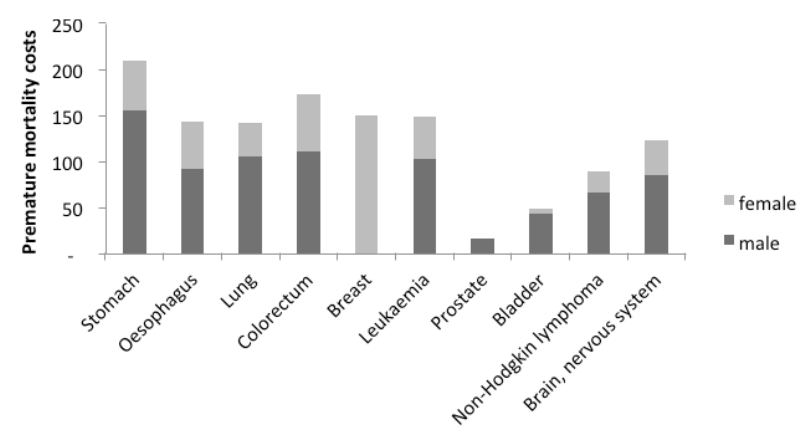

Figure 2. Premature Mortality Costs (US \$ Million) for the 10 Most Common Cancers in Both Sexes (Ranked by Mortality), in Iran 2012 
Table 2. Premature Mortality Costs for the 10 most Common Males and Females Cancers and all Cancers in Iran, 2012, (US \$)

\begin{tabular}{|c|c|c|c|c|c|}
\hline ICD-10 & Cancer site & $\begin{array}{l}\text { Total premature } \\
\text { mortality cost } \\
\text { Discounted at } 3 \%\end{array}$ & $\%$ of the total & $\begin{array}{c}\text { Premature } \\
\text { mortality cost } \\
\text { per death }\end{array}$ & $\begin{array}{l}\text { Premature } \\
\text { mortality cost } \\
\text { per YPLL }\end{array}$ \\
\hline \multicolumn{6}{|c|}{ Males } \\
\hline \multirow[t]{2}{*}{ C00-97/C44 } & All cancers excl. non-melanoma skin cancer & $1,208,963,109$ & 100.00 & 40,946 & 2,313 \\
\hline & Top 10 cancers & $813,116,676$ & 67.26 & 36,586 & 2,181 \\
\hline $\mathrm{C} 15$ & oesophagus & $92,562,780$ & 7.66 & 34,772 & 2,113 \\
\hline $\mathrm{C} 16$ & Stomach & $156,159,040$ & 12.92 & 27,575 & 1,853 \\
\hline C18-21 & Colorectal & $110,584,343$ & 9.15 & 48,931 & 2,532 \\
\hline $\mathrm{C} 22$ & Liver & $33,776,474$ & 2.79 & 40,306 & 2,284 \\
\hline C33-34 & Lung & $105,416,646$ & 8.72 & 35,759 & 2,149 \\
\hline C61 & Prostate & $16,449,135$ & 1.36 & 7,161 & 694 \\
\hline C67 & Bladder & $43,492,747$ & 3.60 & 23,806 & 1,692 \\
\hline $\mathrm{C} 70-72$ & Brain and CNS & $85,867,718$ & 7.10 & 90,103 & 3,216 \\
\hline C82-85,C96 & Non-Hodgkin lymphoma & $66,139,152$ & 5.47 & 56,385 & 2,687 \\
\hline C91-95 & Leukemia & $102,668,639$ & 8.49 & 64,008 & 2,816 \\
\hline \multicolumn{6}{|c|}{ Females } \\
\hline \multirow[t]{2}{*}{ C00-97/C44 } & All cancers excl. non-melanoma skin cancer & $721,876,802$ & 100.00 & 31,720 & 1,402 \\
\hline & Top 10 cancers & $513,352,635$ & 71.11 & 32,400 & 1,415 \\
\hline $\mathrm{C} 15$ & oesophagus & $51,270,207$ & 7.10 & 22,756 & 1,224 \\
\hline C16 & Stomach & $53,761,123$ & 7.45 & 20,838 & 1,170 \\
\hline C18-21 & Colorectal & $62,440,945$ & 8.65 & 31,330 & 1,403 \\
\hline $\mathrm{C} 22$ & Liver & $13,344,245$ & 1.85 & 21,015 & 1,172 \\
\hline C33-34 & Lung & $37,196,386$ & 5.15 & 26,418 & 1,305 \\
\hline C50 & Breast & $149,933,799$ & 20.77 & 45,393 & 1,593 \\
\hline C56 & Ovary & $38,398,286$ & 5.32 & 35,886 & 1,477 \\
\hline $\mathrm{C} 70-72$ & Brain and CNS & $37,660,041$ & 5.22 & 49,553 & 1,621 \\
\hline C82-85,C96 & Non-Hodgkin lymphoma & $23,099,970$ & 3.20 & 30,924 & 1,374 \\
\hline C91-95 & Leukemia & $46,247,634$ & 6.41 & 42,235 & 1,522 \\
\hline
\end{tabular}

stomach cancer ( $\$ 210$ million), followed by colorectal (\$173 million), breast (\$150 million), leukemia (\$149 million) and oesophagus (\$144 million).

\section{Discussion}

This study quantified the burden of cancer in Iran measured by mortality rate, YPLL, and productivity loss. There were 53,350 cancer-related deaths in Iran in $2012,56.4 \%$ of them were in the males. Deaths caused by cancers accounted for 1,112,680 YPLL in total, 50.6\% of them were in the males. The highest number of YPLL among the males and female was related to stomach and breast cancers, respectively. In both sexes combined, the highest number of YPLL was related to stomach cancer. The study estimated that the total productivity costs from premature cancer mortality were approximately US $\$ 1.93$ billion. The most costly cancer for males was stomach; while for females it was breast. In both sexes combined, the most costly cancer was stomach cancer.

As previous studies (Ekwueme et al., 2008; Li and Ekwueme, 2010; Ekwueme et al., 2011), the study used the life expectancy method to calculate the YPLL instead of using an age as cutoff (for example 70 or 75), because this method is more suitable for chronic disease such as cancer that the majority of deaths occur at older ages (Horm and Sondik, 1989). The study findings showed that the average of YPLL per cancer death in the females was more than the males (23.64 versus 18.71). Pham et al in their study in Japan observed that the average years of life lost due to cancer in the females was more than males in 2005 (16.7 years versus 13.9 years) (Pham et al., 2010). They suggested that this difference could be for two reasons: (1) the females' deaths occurred in younger ages than the males, and (2) the life expectancy in the females was longer than the males, thus the YPLL in a specific age was higher in the females than the males. It seems that, these reasons can explain the difference between the males and females in this study, too. for instance, $44 \%$ of the females cancer deaths occurred in ages under 60 , while $30 \%$ of the males cancer deaths occurred in this age group (Ferlay et al., 2012); and the life expectancy at birth in the females was 76 years, while it was 72 years in the males in Iran for the year of 2012 (World Health Organization, 2014).

In this study, as similar to the previous studies, the cost of productivity lost was estimated by the human capital approach (Bradley et al., 2008; Yabroff et al., 2008; Hanly et al., 2012; Hanly et al., 2014). The total productivity costs from premature cancer mortality accounts for approximately $0.034 \%$ of the Iran gross domestic production (GDP; US\$ 551.6 billion) in 2012. Hanly et al concluded the premature mortality costs are considerable amounted to $0.3 \%$ of GDP in Ireland in 2009 (Hanly and Sharp, 2014). Bradley et al found the total cost of productivity from all cancer deaths in the US in 2000 was $\$ 115.8$ billion (Bradley et al., 2008). Hanly et al. (2014) estimated the total premature mortality costs for all cancers were $€ 509.5$ million in Ireland in 2009 (Hanly and 
Sharp, 2014). An important point is that the total number of death from cancer in the US (2010) and Ireland (2009) were 657,005 and 8067 , respectively, while it was 53,350 in Iran in 2012. Comparison of cancer mortality number and the total premature mortality costs in Iran with these two countries shows that premature mortality costs per cancer death in Iran is less than these countries; it might be as a result of the lower employment rate, especially in the females, and the lower wage rate in Iran. According to the World Bank data, Labor force participation rate in 2012 in the US, Ireland and Iran were 63, 60, and 45 percent, respectively (World Bank, 2014).

In this study the total cost of premature mortality for males was $67 \%$ higher than females $(\$ 1,209$ million $v s$. $\$ 722$ million). This difference is due to higher number of death among the males $(30,115$ for males vs. 23,235 foe females in 2012), and higher employment and wage rate for males. The results of previous studies in Ireland and the US showed that the total costs of premature mortality from all cancers were $90 \%$ and $80 \%$ times higher in the males than females, respectively (Bradley et al., 2008; Hanly and Sharp, 2014).

The study result showed that the top 10 ranked cancers accounted for about $70 \%$ of total YPLL and total premature mortality costs in both sexes. The ranking of the top 10 cancer changed based on various measures. For example, among the top 10 sites, Brain and CNS cancer ranked as 1st in terms of cost per death and YPLL per death, while it was ranked as 6th in terms of total costs. In contrast, stomach cancer dropped from 1 st in terms of total costs and YPLL to 8th in terms of cost per death and cost per YPLL. As well as prostate cancer accounted for $7.63 \%$ of the total males cancer deaths, it accounted for $4.21 \%$ and $1.36 \%$ of total males YPLL and premature mortality cost, respectively.

These changes are due to the different ages of death for each cancer which is influenced by the age at diagnosis and survival rate. Cancers with earlier age at the diagnosis and low survival rate usually are placed in the higher rank of YPLL per death and cost per death (Burnet et al., 2005; Hanly and Sharp, 2014). For example, 65\% of death and $75 \%$ of incidence number of brain and CNS in Iran occurred under ages of 60 , while they were $1 \%$ and $11 \%$ for prostate cancer, respectively (Ferlay et al., 2012). Based on these findings, prevention of cancer deaths at the younger ages can reduce the higher cost and YPLL per death than the cancer deaths at the older ages.

According to the study estimations, among males and in the both sexes combined, stomach cancer had the highest number of YPLL and was the most costly cancer in terms of total premature mortality cost. Although the Incidence and mortality rate for stomach cancer have decreased substantially in the most part of the world (Jemal et al., 2010), they have increased in Iran. It is recently the second most commonly diagnosed cancer and the leading cause of cancer death in Iran (Ferlay et al., 2012). Among females breast cancer had the highest number of YPLL (17.15\% from total YPLL) and was the most costly cancer in terms of total premature mortality cost $(21 \%$ from total cost). Breast cancer is the most commonly diagnosed cancer in Iran and the incidence and mortality of this cancer are increasing significantly (Taghavi et al., 2012; Rashidian et al., 2013). These measures indicate that in setting the priorities for cancer control programs in Iran, stomach and breast cancer are the most important ones. In addition, reducing the YPLL and total premature mortality costs due to cancer, the policy makers should consider the specific programs for the prevention and control of these two cancers.

Given the rising trend of the cancer incidence and mortality in LMCs such as Iran (Kanavos, 2006), the YPLL and the premature mortality cost due to cancer will increase in LMCs in future. The estimations in this study highlight the importance of cancer control programs and can be used by the Policy makers to set priority of resource allocation for the health care programs and specially cancer control interventions.

Strength and limitation: As the study compilers have investigated, this is the first national study to estimate the YPLL and cost of productivity lost due to cancer-related premature mortality for an Eastern Mediterranean country. Different measures that used in this study are complementary and provide the policy makers a comprehensive insight to burden of cancer in Iran. Nevertheless, this study has some limitations. First, the study used the human capital approach to estimate the cost of productivity lost. In this approach the value of human life is based on market earnings, so the older males who are working in comparison to the younger males and females, who earn less, are assigned as a higher value. Although there are the alternative approaches such as willingness to pay and friction cost, these approaches are not without limitations (Tarricone, 2006). Second, because there was not data on wage rate of housekeeping activity and its value for the females, the study used the minimum daily wage in 2012 (\$10.5) (Ministry of labor and social Welfare, 2013). So, the study findings about the females cost cancer may be different from the actual cost.

In conclusion, the study indicates that the economic burden of cancer- related premature mortality is substantial, amounting approximately $\$ 2$ billion in Iran for 2012 and is expected to increase in future. The overall cost of productivity lost for the males was 1.67 times higher than the females. Also, stomach, brain and CNS cancers were the most costly in terms of total cost and cost per death. Quantifying the burden of cancer on society can be used by the health policy makers to decide how health resources should be allocated among and between different programs. It should be also mentioned that investments in programs that reduce stomach, breast, lung, and colorectal cancers mortality can be likely to yield the largest annual reduction in productivity costs for Iran society.

\section{References}

Abachizadeh K, Mohagheghi M-A, Mosavi-Jarrahi A (2011). Setting research priorities to reduce burden of cancer in Iran: an institutional experience. Asian Pac J Cancer Prev, 12, 2365-70.

Akbarzadeh Ba, Esmaeili M, Kimiafar K (2008). Medical information management and assessment of direct costs of treatment of lung cancer. Health Information Management, 
Soheila Khorasani et al 5, 151-8 (in Persian).

Bazyar M, Pourreza A, Harirchi I, et al (2010). Medical and non-medical direct costs of cancers in patients hospitalized in Imam Khomeini Cancer Institution-2010. Hospital, 11, 39-50 (in Persian).

Bradley CJ, Yabroff KR, Dahman B, et al (2008). Productivity costs of cancer mortality in the United States: 2000-2020. J Natl Cancer Inst, 100, 1763-70.

Burnet N, Jefferies S, Benson R, et al (2005). Years of life lost (YLL) from cancer is an important measure of population burden-and should be considered when allocating research funds. Br J Cancer, 92, 241-5.

D'Souza ND, Murthy NS, Aras RY (2013). Projection of cancer incident cases for India -till 2026. Asian Pac J Cancer Prev, 14, 4379-86.

Davari M, Yazdanpanah F, Aslani A, et al (2013). The direct medical costs of breast cancer in Iran: analyzing the patient's level data from a cancer specific hospital in Isfahan. Int $J$ Prev Med, 4, 748.

Ekwueme DU, Chesson HW, Zhang KB, et al (2008). Years of potential life lost and productivity costs because of cancer mortality and for specific cancer sites where human papillomavirus may be a risk factor for carcinogenesisUnited States, 2003. Cancer, 113, 2936-45.

Ekwueme DU, Guy Jr GP, Li C, et al (2011). The health burden and economic costs of cutaneous melanoma mortality by race/ethnicity-United States, 2000 to 2006. J Am Acad Dermatol, 65, 133-43

Ferlay J, Soerjomataram I, Ervik M, et al (2012). GLOBOCAN 2012 v1.0, Cancer Incidence and Mortality Worldwide: IARC Cancer Base No. 11 [Internet]. Lyon, France: International Agency for Research on Cancer; 2013. Available from: http:// globocan.iarc.fr, accessed on 10/10/2014. [Online].

Gardner JW, Sanborn JS (1990). Years of potential life lost (YPLL)-what does it measure? Epidemiol, 1, 322-9.

Hanly P, Pearce A, Sharp L (2014). The cost of premature cancerrelated mortality: a review and assessment of the evidence. Expert review of pharmacoeconomics and outcomes research, 1-23.

Hanly P, Soerjomataram I, Sharp L (2015). Measuring the societal burden of cancer: The cost of lost productivity due to premature cancer-related mortality in Europe. Int $J$ Cancer, 136, 136-45.

Hanly P,Timmons A, Walsh PM, et al (2012). Breast and prostate cancer productivity costs: a comparison of the human capital approach and the friction cost approach. Value Health, 15, 429-36.

Hanly PA, Sharp L (2014). The cost of lost productivity due to premature cancer-related mortality: an economic measure of the cancer burden. BMC Cancer, 14, 224.

Horm JW, Sondik EJ (1989). Person-years of life lost due to cancer in the United States, 1970 and 1984. Am J Public Health, 79, 1490-3.

Iran SCo (2013). Iran Statistical Yearbook, available from: http://www.amar.org.ir/Default.aspx?tabid=2153, accessed on:(10.09.2013) [Online].

Jemal A, Center MM, DeSantis C, et al (2010). Global patterns of cancer incidence and mortality rates and trends. Cancer Epidemiol Biomarkers Prev, 19, 1893-907.

Jonsson B, Wilking N (2007). The burden and cost of cancer. Ann Oncol, 18, 8-22.

Kanavos P(2006). The rising burden of cancer in the developing world. Ann oncol, 17, 15-23.

Kim JL, Cho KH, Park EC, et al (2014). A single measure of cancer burden combining incidence with mortality rates for worldwide application. Asian Pac J Cancer Prev, 15, 433-9.

Lee WC (1997). Quantifying the future impact of disease on society: life table-based measures of potential life lost. Am J Public Health, 87, 1456-60.

Li C, Ekwueme DU (2010). Years of potential life lost caused by prostate cancer deaths in the United States-projection from 2004 through 2050. Cancer Epidemiol, 34, 368-72.

Ministry of Labour and Social Welfare, Iran (2013). Labour Market Information System. available from: Http://www. Amarkar.Ir/asp/statreport.Aspx. accessed on:(01/04/2013) [Online].

Naghavi M, Abolhassani F, Pourmalek F, et al (2009). The burden of disease and injury in Iran 2003. Population Health Metrics, 7, 9 .

Pham TM, Fujino Y, Matsuda S, et al (2010). Premature mortality due to cancer in Japan, 1995 and 2005. Int J Cancer, 127, 190-4.

Rashidian H, Davoudi R, Ghiasvand R, et al (2013). Prevalence and Incidence of premenopausal and postmenopausal breast cancer in Iran in 2010. Basic Clinical Cancer Res, 5, 2-10.

Roya N, Abbas B (2013). Colorectal cancer trends in Kerman province, the largest province in Iran, with forecasting until 2016. Asian Pac J Cancer Prev, 14, 791-3.

Taghavi A, Fazeli Z, Vahedi M, et al (2012). Increased trend of breast cancer mortality in Iran. Asian Pac J Cancer Prev, 13, 367-70.

Tarricone R (2006). Cost-of-illness analysis: What room in health economics? Health Policy, 77, 51-63.

The central bank of Iran (CBI). 2014. exchange rate, available at: Http://www.Cbi.Ir/exrates/rates_fa.Aspx, accessed on: (20.09.2014) [Online].

World Bank (2014). World Development Indicators, Labor force participation rate, available at: http://data.worldbank. org/indicator/SL.TLF.CACT.ZS ,accessed on:(10.10.2014).

World Health Organization. 2014. Global Health Observatory Data Repository ; available from: http://apps.who.int/gho/ data/?theme $=$ mainand vid $=60760$, accessed on: $(01 / 10 / 2014)$ [Online].

Yabroff KR, Bradley CJ, Mariotto AB, et al (2008). Estimates and projections of value of life lost from cancer deaths in the United States. J Natl Cancer Inst, 100, 1755-62. 